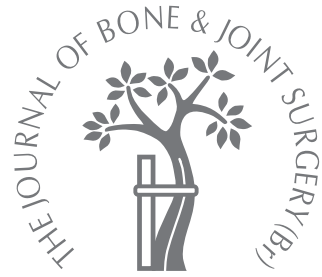

J. P. Strickland, J. W. Sperling, R. H. Cofield

From the Mayo Clinic, Rochester, Minnesota, United States
J. P. Strickland, MD, Resident I. J. W. Sperling, MD, MBA, Associate Professor I. H. Cofield, MD, Professor Mayo Clinic, 200 First Street SW, Rochester, Minnesota 55905, USA.

Correspondence should be sent to Dr J. W. Sperling; e-mail: sperling.john@mayo.edu

(C)2008 British Editorial Society of Bone and Joint Surgery doi:10.1302/0301-620X.90B4 $20002 \$ 2.00$

$J$ Bone Joint Surg $[\mathrm{Br}]$ 2008;90-B:460-5.

Received 25 July 2007;

Accepted after revision 22 November 2007

\title{
The results of two-stage re-implantation for infected shoulder replacement
}

\author{
While frequently discussed as a standard treatment for the management of an infected \\ shoulder replacement, there is little information on the outcome of two-stage \\ re-implantation.
}

We examined the outcome of 17 consecutive patients (19 shoulders) who were treated between 1995 and 2004 with a two-stage re-implantation for the treatment of a deepinfection after shoulder replacement. All 19 shoulders were followed for a minimum of two years or until the time of further revision surgery. The mean clinical follow-up was for 35 months (24 to 80 ). The mean radiological follow-up was 27 months (7 to 80 ). There were two excellent results, four satisfactory and 13 unsatisfactory. In 12 of the 19 shoulders $(63 \%)$ infection was considered to be eradicated. The mean pain score improved from 4.2 (3 to 5 (out of 5$)$ ) to $1.8(1$ to 4$)$. The mean elevation improved from $42^{\circ}\left(0^{\circ}\right.$ to $\left.140^{\circ}\right)$ to $89^{\circ}\left(0^{\circ}\right.$ to $\left.165^{\circ}\right)$, mean external rotation from $30^{\circ}\left(0^{\circ}\right.$ to $\left.90^{\circ}\right)$ to $43^{\circ}\left(0^{\circ}\right.$ to $\left.90^{\circ}\right)$, and mean internal rotation from the sacrum to L5. There were 14 complications.

Our study suggests that two-stage re-implantation for an infected shoulder replacement is associated with a high rate of unsatisfactory results, marginal success at eradicating infection and a high complication rate.

Infection after shoulder replacement is a rare but devastating complication. The reported incidence for unconstrained primary shoulder replacement ranges from $0 \%$ to $4 \% .^{1-4}$ However, there is little published information available on the treatment of the infected shoulder prosthesis. $^{1,2,5-9}$ The outcome after resection arthroplasty is poor although the shoulder is usually comfortable at rest. ${ }^{10}$

Two-stage re-implantation has become an accepted treatment for infected hip and knee replacements. ${ }^{11,12}$ The purpose of this paper is to describe the results, risk factors for unsatisfactory outcome, and rate of failure following two-stage re-implantation in the treatment of an infected shoulder replacement.

\section{Patients and Methods}

The patients were identified from our total joint registry that prospectively maintains records on all patients who have undergone a total joint replacement at our hospital since 1969. Between 1995 and 2004, 1349 patients (1511 shoulders) underwent primary shoulder replacement and 287 patients (316 shoulders) underwent revision shoulder replacement. The study was approved by our institutional review board. During this period 17 patients (19 shoulders) were treated with a two-stage reimplantation for the treatment of a deep infection after shoulder replacement. The initial diagnoses which led to the primary replacement were osteoarthritis in ten patients (11 shoulders), rheumatoid arthritis in one (two shoulders), fracture in five (five shoulders) and avascular necrosis in one (one shoulder). The initial surgery comprised 11 total shoulder replacements (nine glenoid and two humeral components were cemented), and eight humeral head replacements (four were cemented). Of the primary procedures, seven were performed at our hospital and 12 were performed elsewhere. The mean follow-up from the time of re-implantation was for 35 months (24 to 80 ).

There were ten men and seven women with a mean age of 62 years ( 41 to 75 ). At the time of this study, one patient had died due to unrelated causes. Data from the last examination was used for this patient. There were 13 right shoulders and six left. All patients presented with a painful shoulder replacement and a suspicion of an infection. Two patients presented with a concomitant peri-prosthetic fracture; one had an acute fracture and the other a humeral nonunion. 
Table I. Classification of infection

\begin{tabular}{lll}
\hline Classification & $\begin{array}{l}\text { Time after initial replacement } \\
\text { (mths) }\end{array}$ & $\begin{array}{l}\text { Number of } \\
\text { shoulders }\end{array}$ \\
\hline Acute & $<3$ & 3 \\
Subacute & 3 to 12 & 7 \\
Late & $>12$ & 9 \\
\hline
\end{tabular}

No identifiable risk factors for infection could be identified in nine patients (nine shoulders). Two patients (three shoulders) were immunosuppressed on prednisolone, three were diabetic (three shoulders) and one (one shoulder) had chronic lymphoedema. In three patients (four shoulders) previous treatment for infection of the shoulder prosthesis had failed to eradicate infection, this included long-term antibiotic suppression in one (two shoulders), three arthroscopic debridements in one (one shoulder) and open debridement in one (one shoulder).

The diagnosis of infection was classified as acute, subacute or late (Table I). There were three acute, seven subacute and nine late infections. The interval from the initial shoulder replacement to the diagnosis of infection was a mean of 35 months (1 to 156 ).

Pre-operative data including pain, range of movement, and signs and symptoms of infection were recorded. Pain was recorded on a scale ranging from 1 to 5 points, in which 1 indicated no pain, 2 mild pain, 3 moderate pain after unusual activity, 4 moderate pain and 5 severe pain. The investigations for infection included full blood count, erythrocyte sedimentation rate (ESR), C-reactive protein (CRP), pre-operative aspiration in nine patients and indium-labelled white blood cell (WBC) scans in eight. Two patients (two shoulders) were referred for re-implantation after resection arthroplasty and placement of an antibioticimpregnated spacer performed elsewhere. All 15 patients (17 shoulders) who had resection performed at our unit had intra-operative frozen-section pathology and biopsies for microbiological culture. In four patients (four shoulders) the cultures failed to identify an organism. However, both the pre- and intra-operative findings were consistent with infection in these patients. In addition, these four patients met the criteria previously reported by Spangehl et $\mathrm{al}^{13}$ for diagnosing an infected hip replacement. The mean time from onset of symptoms to resection for all 19 shoulders was 9.5 months ( 2 days to 28 months).

An antibiotic spacer was implanted at the time of the resection in all 15 patients (17 shoulders) (Fig. 1). This contained a combination of vancomycin and gentamicin in 14 shoulders and vancomycin and tobramycin in one shoulder. The two patients (two shoulders) who underwent resection elsewhere had antibiotic spacers implanted but it was not known which antibiotics were used. All patients were seen and evaluated in consultation with a microbiologist with a special interest in orthopaedics. All patients received intravenous antibiotics specific to the organism being treated for

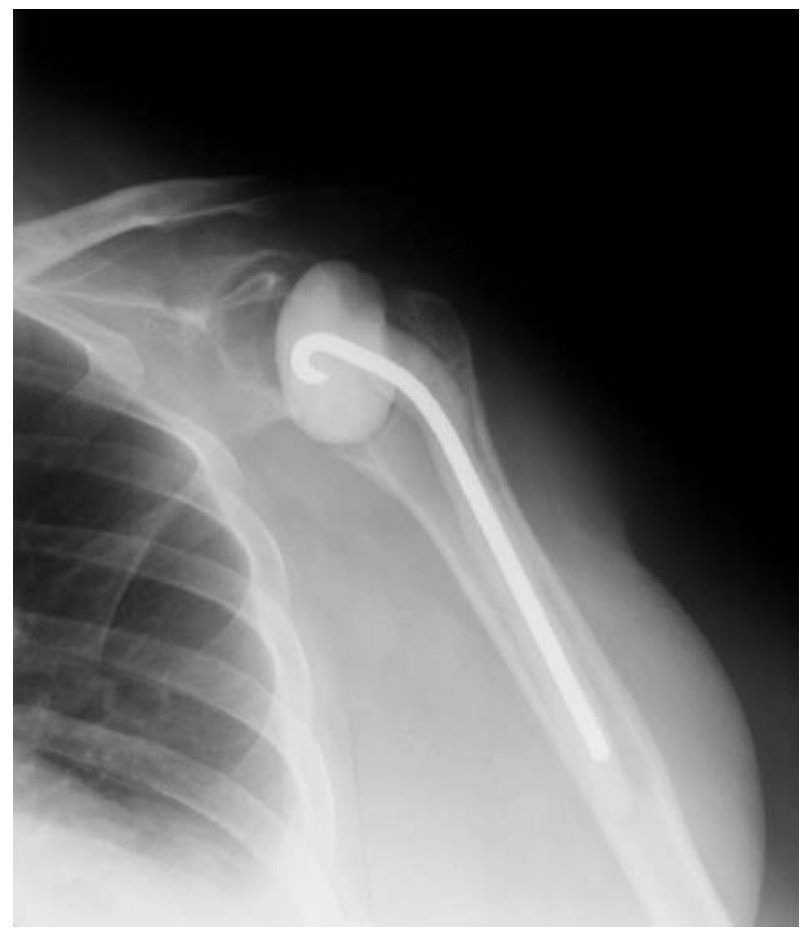

Fig. 1

Immediate post-operative anteroposterior radiograph of the left shoulder following removal of infected components and implantation of an antibiotic cement spacer.

four to six weeks between resection and re-implantation. Of the four patients with negative intra-operative cultures, three received intravenous cefazolin for six weeks and one patient received intravenous ceftriaxone for four weeks.

The mean time from resection to re-implantation was 11 weeks ( 6 to 31). At the time of re-implantation all patients had a full blood count, ESR and 16 of 17 patients had a CRP. One patient had an aspiration performed before the reimplantation which was negative. Further indium-labelled WBC scans were not performed prior to re-implantation as they were all negative at the time of resection. All patients had intra-operative pathology and biopsies for microbiological culture taken at the time of re-implantation. Post-operative pain, range of movement, and the Neer rating ${ }^{14}$ was recorded for all patients. The result was considered excellent if the patient had no or slight pain, active abduction to $140^{\circ}$, and external rotation to $45^{\circ}$, and was satisfied with the procedure. A satisfactory result consisted of no or slight pain or moderate pain only with vigorous activity, active abduction to $90^{\circ}$, external rotation to $20^{\circ}$, and the patient being satisfied with the procedure. A result was graded as unsatisfactory if the criteria for satisfaction were not met or if the patient needed a further revision procedure. Any patient who required chronic antibiotic suppression was considered to be still infected and given an unsatisfactory rating. All complications including re-operation were recorded. 


\begin{tabular}{ll} 
Table II. Grading of peri-prosthetic radiolucencies & \\
\hline Grade & \\
\hline 0 & No lines \\
1 & $1 \mathrm{~mm}$ wide and incomplete \\
2 & $1 \mathrm{~mm}$ wide and complete \\
3 & $1.5 \mathrm{~mm}$ wide and incomplete \\
4 & $1.5 \mathrm{~mm}$ wide and complete \\
5 & $2 \mathrm{~mm}$ wide and complete \\
\hline
\end{tabular}

A radiological assessment was also performed. The mean radiological follow-up was 27 months (7 to 80) following re-implantation. Routine radiographs included a $40^{\circ}$ posterior oblique view with the humerus in both internal and external rotation in addition to an axillary view of the shoulder. Glenohumeral subluxation was determined by the amount and direction of translation of the humeral head in relation to the centre of the glenoid component. Subluxation was recorded as none, as mild if there was $<25 \%$ translation, as moderate if there was $26 \%$ to $50 \%$ translation, and as severe if there was $>50 \%$ translation.

Peri-prosthetic radiolucency was graded according to the scale of Mileti, Sperling and Cofield ${ }^{15}$ (Table II). Migration of the glenoid as well as subsidence of the humeral component was recorded.

Statistical analysis. The associations between pre-operative and post-operative pain, elevation, internal rotation and external rotation were assessed with paired $t$-tests. A p-value $<0.05$ was considered significant.

\section{Results}

Resection. At the time of resection the CRP was elevated in eight of the 16 shoulders (14 patients) tested. The ESR was elevated in eight of 17 shoulders (15 patients) tested. The WBC was normal in all shoulders at the time of resection. Pre-operative aspiration was positive in six of nine shoulders (eight patients). All shoulders with a negative preoperative aspiration had positive tissue cultures obtained at the time of surgery. Indium-labelled WBC scans were obtained in eight shoulders (seven patients) and all were negative.

In four of the 17 shoulders who had resection performed at our hospital a humeral osteotomy was required for implant removal. The results of the tissue cultures were available in 18 shoulders (16 patients). This was not available in one patient resected at an outside facility. No patient was receiving antibiotic treatment at the time of resection. Culture at the time of surgery did not reveal an organism in four shoulders. In ten shoulders (ten patients) the organism was either Proprionibacterium acnes or coagulase-negative Staphylococcus. Three shoulders were infected with Staphylococcus aureus and one shoulder grew Enterococcus. One shoulder which grew Proprionibacterium acnes had a raised CRP with a normal ESR and one

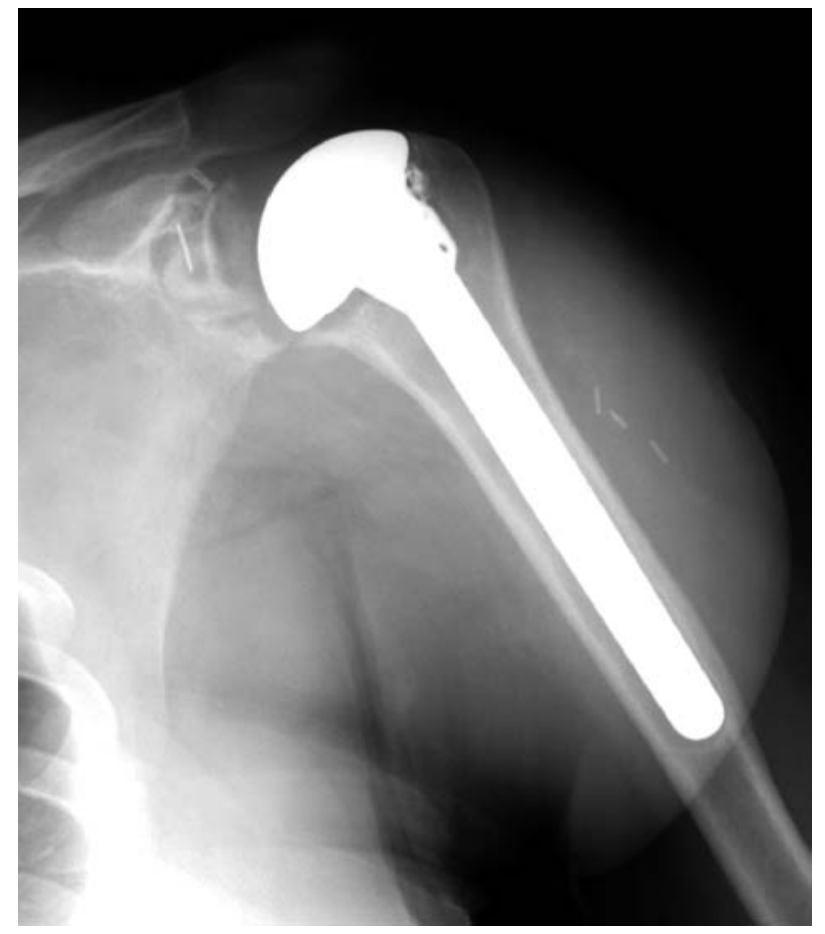

Fig. 2

Immediate pre-operative anteroposterior radiograph of an infected left shoulder replacement with evidence of medial calcar erosion and endosteal erosion of the humerus with a completely displaced glenoid component 11 years after primary total shoulder replacement.

with the same organism had an elevated pre-operative ESR with a normal CRP.

Of the 16 shoulders (15 patients) with pre-operative radiographs available, eight $(50 \%)$ had evidence of humeral radiolucencies at the time of resection. Of the nine shoulders with a cemented glenoid component pre-operatively there were associated radiolucencies in eight. Three glenoid components were displaced (Fig. 2). In ten shoulders $(63 \%)$ there was an erosion of the medial calcar, and in four $(25 \%)$ there was reabsorption of bone at the tuberosities. Mild glenohumeral subluxation was present in four patients and moderate subluxation had occurred in three.

Re-implantation. At the time of re-implantation eight shoulders (six patients) had an elevated CRP and seven (seven patients) an elevated ESR, but in all shoulders the WBC was within normal limits. Intra-operative histology showed no evidence of acute inflammation in all shoulders. In three shoulders (three patients) positive intra-operative microbiological cultures were obtained. Two of these patients received long-term antibiotic treatment and one had treatment for four weeks after re-implantation.

For re-implantation a deltopectoral approach was used in 14 shoulders and an anteromedial approach in five. A Cofield hemiarthroplasty (Smith \& Nephew, Memphis, Tennessee) was used in 13 shoulders and total shoulder replacement was used for five shoulders (four Cofield and 


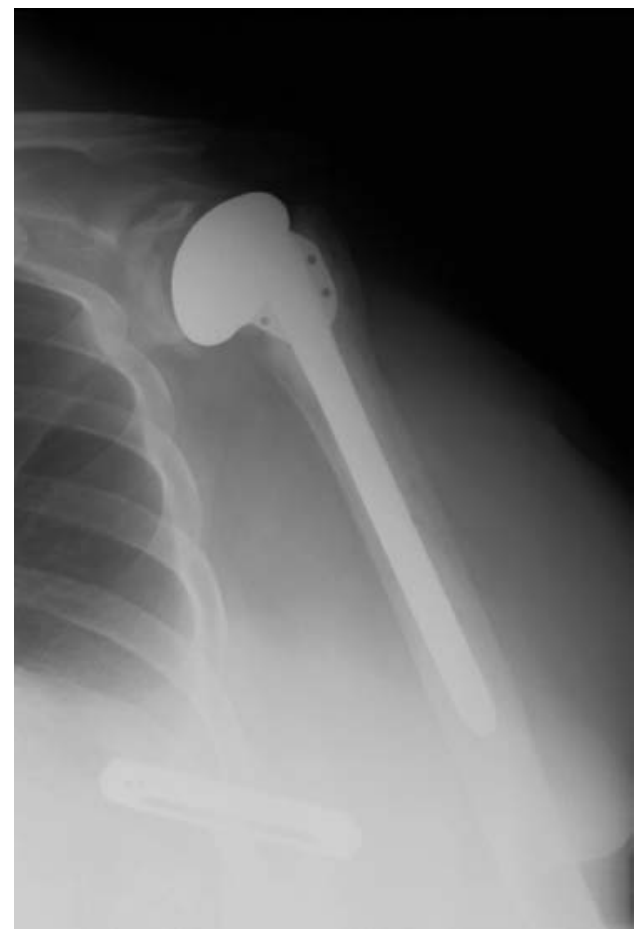

Fig. 3

Anteroposterior radiograph of the left shoulder 27 months after revision with implantation of a humeral hemiarthroplasty.

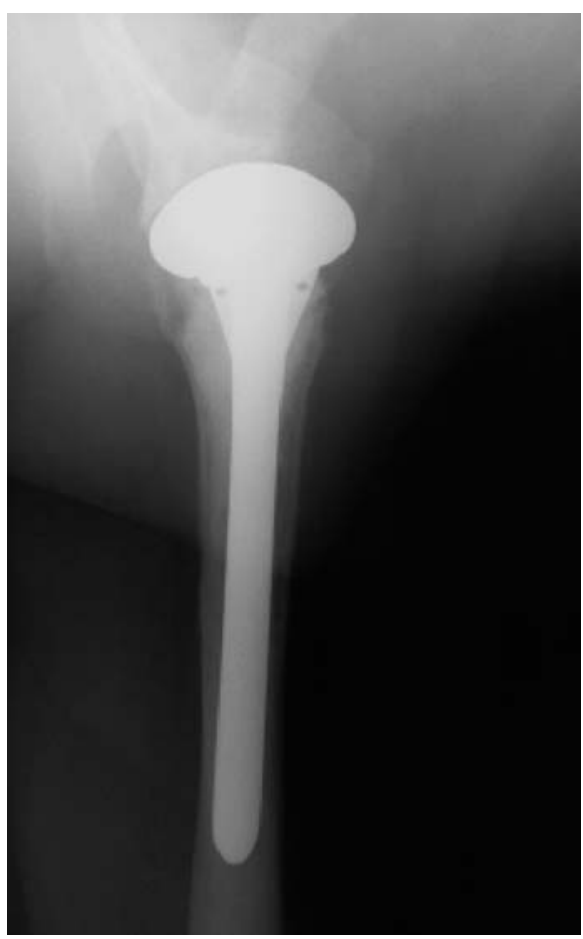

Fig. 4

Lateral radiograph of a revision left humeral hemiarthroplasty, 27 months after re-implantation. one Aequalis; Tornier, Grenoble, France and one Delta III reverse prosthesis; Depuy, Warsaw, Indiana). Of 13 shoulders with a cemented humeral component, 12 had antibiotic-laden cement (Figs 3 and 4). In five shoulders a long-stem component was required due to proximal bone loss.

Post-operative radiographs were available in all patients. Seven of 18 shoulders had evidence of humeral radiolucencies at the most recent follow-up. One of the six shoulders with a glenoid component had a $1.5 \mathrm{~mm}$ wide incomplete lucency at the cement-bone interface. Mild glenohumeral subluxation was present in eight shoulders, two had moderate subluxation, four were considered to have severe subluxation, and three had no subluxation. There was no evidence of humeral subsidence in any shoulder.

The mean pre-operative elevation was $42^{\circ}\left(0^{\circ}\right.$ to $\left.140^{\circ}\right)$, mean external rotation was $30^{\circ}\left(0^{\circ}\right.$ to $\left.90^{\circ}\right)$ and the mean internal rotation to the level of the sacrum (abdomen to T7). The mean pre-operative pain score was 4.2 (3 to 5 ) on the five point scale.

The mean post-operative elevation of the shoulder was $89^{\circ}\left(0^{\circ}\right.$ to $\left.165^{\circ}\right)$, the mean external rotation was $43^{\circ}\left(0^{\circ}\right.$ to $90^{\circ}$ ) and the mean internal rotation was to L5 (ilium to T7). When compared with the pre-operative ranges there was a significant improvement in elevation $(\mathrm{p}=0.003)$ but not for external or internal rotation $(\mathrm{p}=0.15$ and $\mathrm{p}=0.1$, respectively). Post-operatively the mean pain score was 1.8 (1 to 4). This represented a significant improvement compared with the mean pre-operative score of 4.2 $(\mathrm{p}=0.0001)$. The outcome was excellent in two shoulders, satisfactory in four and unsatisfactory in 13 , according to the rating scale of Neer. ${ }^{14}$ The two excellent outcomes were in one of our two patients with bilateral infected shoulder replacements.

Complications. A total of 14 complications occurred. At the time of resection, there were two fractures of the humerus. At the time of re-implantation, there was one fracture of the greater tuberosity, a post-re-implantation subscapularis tear and associated haematoma, a humeral nonunion, an unstable implant, and continued chronic pain in one patient who underwent a humeral head reimplantation. There were two non-fatal pulmonary emboli. Antibiotic-related complications occurred in three patients including hyperpigmentation in one, severe gastrointestinal intolerance in another, and one patient developed severe Clostridium difficile colitis. One patient had persistent post-operative wound drainage and eventually underwent resection arthroplasty.

A total of five further operations followed re-implantation including two open irrigation and debridements, open reduction and internal fixation of a fracture of the greater tuberosity, implantation of a glenoid component in the 
patient with chronic pain, and resection arthroplasty in the patient with continued infection.

Five patients (six shoulders) were treated with long-term antibiotics, of whom two had a positive culture at the time of re-implantation. One patient had positive cultures at fixation of his greater tuberosity fracture and two were elderly and further surgery was contraindicated. If all patients on long-term antibiotics and the patient who had a resection arthroplasty were considered to be infected, successful eradication of infection was achieved in 12 of the 19 shoulders $(63 \%)$.

\section{Discussion}

Diagnosing infection in patients requiring a revision of a shoulder replacement can be difficult as many lack overt signs of infection such as erythema or sinus tracts. Topolski et $\mathrm{al}^{16}$ recently described the value of pre-operative studies and intra-operative histology in patients who underwent revision shoulder replacement and had positive intraoperative cultures. In a series of 75 shoulders without obvious signs of infection undergoing revision the pre-operative WBC count was normal in 67 of 72, with normal polymorphonuclear cell distributions and a normal ESR in most patients. Among the 16 patients who had a CRP measured, it was normal in 12 . The intra-operative histology showed no signs of acute inflammation in 67 of 73 patients. The most commonly-cultured organism was Proprionibacterium acnes in 45 of 75 cases $(60 \%)$. The mean time for the first growth was 5.1 days. The authors concluded that there were no reliable intra- or pre-operative studies to forecast who was likely to have a positive intra-operative culture.

It is wise to consider the possibility of a low-grade infection particularly if there are radiolucencies on pre-operative radiographs. In our study $50 \%$ of the patients had radiolucencies in relation to the humeral component and $89 \%$ (8 of 9) had radiolucencies in the presence of a glenoid component.

Another subtle radiological finding was the presence of medial calcar erosion, and resorption of the tuberosities should also be noted.

Indium-scans did not appear to contribute to the preoperative assessment as they were negative in all of our patients where they were obtained. This is in contrast to the study of Coste et $\mathrm{al}^{2}$ where it was positive in eight of 11 patients.

The literature is scant regarding the outcome following two-stage re-implantation for infection. ${ }^{1,2,5-7}$ Jerosch and Schneppenheim ${ }^{5}$ described eight patients who underwent two-stage exchange and replacement of the humeral head. They reported no evidence of re-infection and a mean postoperative Constant score of 48 . Seitz and Damacen ${ }^{6}$ studied eight patients, five of whom had a previous implant, who underwent staged exchange. The mean post-operative University of Pennsylvania Shoulder score was 68.4 of 100 . The mean subset score for satisfaction was 6 of 10. However, all eight showed significant limitation in movement and strength compared with the opposite side. Antibiotic spacers were recommended in both these papers ${ }^{5,6}$ to maintain soft-tissue tension and facilitate re-implantation. Finally, Coste et $\mathrm{al}^{2}$ reviewed ten patients who underwent two-stage exchange for infection. The mean Constant score rose from 15 to 35 .

It is a concern that seven of our 19 shoulders ( $37 \%$ ) had persistent infection or at least required chronic suppressive antibiotic treatment. This finding is consistent with Coste et al's study ${ }^{2}$ in which $40 \%$ of patients had evidence of ongoing infection despite using a two-stage approach.

The rate of complications was high. Two patients $(11.7 \%)$ had a pulmonary embolism which is a rare complication of shoulder replacement. ${ }^{17}$ Sperling and Cofield ${ }^{18}$ identified five of 2885 patients who underwent primary shoulder replacement between 1981 and 2001 who sustained a pulmonary embolism $(0.17 \%)$. Lyman et al ${ }^{19}$ found the frequency of pulmonary embolism to be 2.3 per 1000 shoulder replacements. The increased incidence in our patients could be related to multiple operations, decreased mobility of the patients following surgery or a chance finding in this small patient population.

The limitations of this study include those inherent in a retrospective review and our patient population was small. In four of our patients no organism was cultured at the time of resection but they were considered infected as they met criteria previously described by Spangehl et al ${ }^{13}$ for infected total hip replacement. Jerosch and Schneppenheim ${ }^{5}$ only identified organisms in four of 12 patients operated on for infection. Coste et $\mathrm{al}^{2}$ could only administer specific antibiotic treatment to 30 of their 42 patients $(71 \%)$ with infected shoulder replacements.

In conclusion, an infected shoulder replacement is difficult to diagnose and treat. In spite of reduced pain and improved active elevation, infection continues in many patients.

The author or one or more of the authors have received or will receive benefits for personal or professional use from a commercial party related directly or indirectly to the subject of this article. In addition, benefits have been or will be directed to a research fund, foundation, educational institution, or other nonprofit organisation with which one or more of the authors are associated.

\section{References}

1. Sperling JW, Kozak TK, Hanssen AD, Cofield RH. Infection after shoulder arthroplasty. Clin Orthop 2001;383:206-16.

2. Coste JS, Reig S, Trojani C, et al. The management of infection in arthroplasty of the shoulder. J Bone Joint Surg [Br] 2004;86-B:65-9.

3. Boileau P, Sinnerton RJ, Chuinard C, Walch G. Arthroplasty of the shoulder. J Bone Joint Surg [Br] 2006;88-B:562-75.

4. Haines JF, Trail IA, Nuttall D, Birch A, Barrow A. The results of arthroplasty in osteoarthritis of the shoulder. J Bone Joint Surg [Br] 2006;88-B:496-501.

5. Jerosch J, Schneppenheim M. Management of infected shoulder replacement. Arch Orthop Trauma Surg 2003;1123:209-14.

6. Seitz WH Jr, Damacen H. Staged exchange arthroplasty for shoulder sepsis. J Arthroplasty 2002:17:36-40.

7. Ramsey ML, Fenlin JM Jr. Use of an antibiotic-impregnated bone cement block in the revision of an infected shoulder arthroplasty. J Shoulder Elbow Surg 1996;5:47982.

8. Loebenberg MI, Zuckerman JD. An articulating interval spacer in the treatment of an infected total shoulder arthroplasty. J Shoulder Elbow Surg 2004;13:476-8. 
9. Ince A, Seemann K, Frommelt L, Katzer A, Loehr JF. One stage exchange shoulder arthroplasty for peri-prosthetic infection. J Bone Joint Surg [Br]2005;87-B:814-18.

10. Rispoli DM, Sperling JW, Athwal GS, Schleck CD III, Cofield RH. Pain relief and functional results after resection arthroplasty of the shoulder. J Bone Joint Surg [Br] 2007;89-B:1184-7.

11. Leone JM, Hanssen AD. Management of infection at the site of a total knee arthroplasty. Instr Course Lect 2006;55:449-61.

12. Haleem AA, Berry DJ, Hanssen AD. Mid-term to long-term followup of two-stage implantation for infected total knee arthroplasty. Clin Orthop 2004;428:35-9.

13. SpangehI MJ, Masri BA, O'Connell JX, Duncan CP. Prospective analysis of preoperative and intraoperative investigations for the diagnosis of infection at the sites of two hundred and two revision total hip arthroplasties. J Bone Joint Surg [Am] 1999;81-A:672-83
14. Neer CS 2nd, Watson KC, Stanton FC. Recent experience in total shoulder replacement. J Bone Joint Surg [Am] 1982;64-A:319-37.

15. Mileti J, Sperling JW, Cofield RH. Reimplantation of a shoulder arthroplasty after a previous infected arthroplasty. J Shoulder Elbow Surg 2004;13:528-31.

16. Topolski MS, Chin PY, Sperling JW, Cofield RH. Revision shoulder arthroplasty with positive intraoperative cultures: the value of preoperative studies and intraoperative histopathology. J Shoulder Elbow Surg 2006;15:402-6.

17. Saleem A, Markel DC. Fatal pulmonary embolism after shoulder arthroplasty. $J$ Arthoplasty 2001;16:400-3.

18. Sperling JW, Cofield RH. Pulmonary embolism following shoulder arthroplasty. J Bone Joint Surg [Am]2002;84-A:1939-41.

19. Lyman S, Sherman S, Carter TI, et al. Prevalance and risk factors for symptomatic thromboembolic events after shoulder arthroplasty. Clin Orthop 2006:448:152-6. 